\title{
Homicídios juvenis e informalidade em um município brasileiro da tríplice fronteira Brasil, Paraguai e Argentina
}

\author{
Luciano de Andrade, ${ }^{1}$ Oscar Kenji Nihei, ${ }^{1}$ Sandra Marisa Pelloso ${ }^{2}$ \\ e Maria Dalva Barros de Carvalho²
}

Como citar Andrade L, Nihei OK, Pelloso SM, Carvalho MDB. Homicídios juvenis e informalidade em um município brasileiro da tríplice fronteira Brasil, Paraguai e Argentina. Rev Panam Salud Publica. 2012:31(5):380-7.

RESUMO Objetivo. Analisar a distribuição espacial da mortalidade juvenil por homicídio para o sexo masculino no Município de Foz do Iguaçu (Estado do Paraná), situado na tríplice fronteira Brasil, Paraguai e Argentina, no período de 2000 a 2007.

Métodos. Os dados foram obtidos no Sistema de Informações sobre Mortalidade (SIM) e no Instituto Brasileiro de Geografia e Estatística (IBGE). Os programas ArcGIS ${ }^{\circledR} 9.2$ e GeoDa ${ }^{T M}$ foram utilizados para analisar 873 óbitos distribuídos em 11 áreas de expansão demográfica (AEDs).

Resultados. Constatou-se uma autocorrelação espacial negativa $(I=-0,3563 ; \mathrm{P}=0,0250)$, demonstrando altas taxas de homicídio em todas as AEDs, mas dissimilares entre si. Dentre 14 diferentes indicadores socioeconômicos, a ocupação no setor informal e a ocupação no setor formal apresentaram autocorrelação espacial negativa $(I=-0,2574 ; \mathrm{P}=0,0360)$ e positiva $(I=0,2574 ; \mathrm{P}=0,0310)$, respectivamente, indicando que quanto maior o número de empregos informais em uma determinada AED menor a taxa de homicídios nas AEDs vizinhas. Identificaram-se nesse estudo altas taxas de homicídios juvenis, ocupação informal e importação de homicídios juvenis de outras AEDs para a AED 6, próxima à fronteira com o Paraguai.

Conclusões. A elevada taxa de homicídios de jovens em algumas regiões do município é influenciada pelo alto grau de ocupação informal, indicando um grave problema social, que éo desemprego associado à falta de qualificação profissional.

Palavras-chave

Violência; homicídio; adolescente; adulto jovem; distribuição espacial da população; Brasil.

O fenômeno da violência juvenil é, atualmente, um problema social de forte impacto. Atinge todas as nações do mundo, embora a sua intensidade varie de

\footnotetext{
Universidade Estadual do Oeste do Paraná (Unioeste), Grupo de Pesquisa em Saúde Coletiva, Centro de Educação e Letras, Foz do Iguaçu (PR), Brasil. Correspondência: Luciano de Andrade, luc.and1973@gmail.com

2 Universidade Estadual de Maringá (UEM), Centro Ciências da Saúde, Maringá (PR), Brasil.
}

continente para continente; afeta particularmente os países de renda baixa e média, com taxas de homicídio de jovens superiores a 10/100 000 (1-4). Em geral, as taxas de homicídio juvenil vêm crescendo, flutuando de 0,6 a 0,9/100 000 habitantes nos países de alta renda da Europa e partes da Ásia e do Pacífico, até 17,6 a 36,4/100 000 habitantes em países com baixa renda e/ ou em desenvolvimento (2).
No Brasil, no período de 1996 a 2006, os homicídios juvenis cresceram 31,3\%, de 13186 para 17312 casos. Assim, a taxa de homicídios na faixa etária de 15 a 24 anos apresentou um aumento superior ao da população total (aumento de 20\%) no mesmo período (5). A taxa mais baixa de homicídios juvenis em 2006 ocorreu na Região Sul do Brasil (40,7/100 000). Entre os três estados que compõem essa 
região, o Estado do Paraná foi o que apresentou a maior taxa de homicídios $(60,3 / 100$ 000) (6).

A Cidade de Foz do Iguaçu, localizada no Estado do Paraná, na região da tríplice fronteira (Brasil, Paraguai e Argentina), conhecida por suas atrações turísticas, tem-se destacado pelas altas taxas de homicídios juvenis. O mapa da violência dos municípios brasileiros (5) mostrou que esse município, no ano de 2006, teve a maior taxa média de homicídios da população jovem, com 234,8 casos em 100000 jovens (5).

Diversos estudos mostram que os jovens do sexo masculino, na faixa etária dos 15 aos 24 anos, são as maiores vítimas da violência (3, 7-9). Além disso, há evidências de que as elevadas taxas de homicídios juvenis correlacionamse com altos níveis de desigualdade socioeconômica $(1,2,7)$.

Uma das formas de procurar compreender e localizar o fenômeno da violência juvenil é por meio da análise espacial $(10,11)$. Os avanços das técnicas de geoprocessamento tornaram possível uma visualização espacial dos homicídios, de forma pontual ou agregada por áreas, permitindo uma análise da população exposta de acordo com diferentes variáveis, assim como o estabelecimento de estratégias de prevenção da violência (12). Diversos estudos utilizaram esse tipo de análise para identificar os locais com maior número de mortes, subsidiando de forma mais efetiva e eficaz a formulação de políticas de intervenção intersetorial (13) e indicando regiões onde as populações deveriam receber atenção especial $(14,15)$.

Este trabalho tem por objetivo analisar a distribuição espacial da mortalidade por homicídio em jovens de 15 a 24 anos no Município de Foz do Iguaçu entre os anos de 2000 e 2007.

\section{MÉTODOS}

Foi realizado um estudo ecológico, descritivo, de corte transversal, utilizando técnicas de análise espacial, com dados secundários do período de 2000 a 2007 referentes aos homicídios de jovens do sexo masculino de 15 a 24 anos em Foz do Iguaçu.

Foz do Iguaçu possui área total de $611,57 \mathrm{~km}^{2}$, com predominância urbana de $99,22 \%$ (16). No ano de 2000, a população de jovens do sexo masculino, de 15 a 24 anos, era de 11989 habitantes, tendo crescido para 13276 em 2004 e 15188 em 2007 (17).

O município é dividido em 261 setores censitários. Para melhor detalhamento dos seus dados socioeconômicos e demográficos, o Instituto Brasileiro de Geografia e Estatística (IBGE) agrupou esses setores em áreas de expansão demográfica (AED), que correspondem à menor unidade de análise territorial dos resultados do censo demográfico de 2000 (18). O Município de Foz do Iguaçu foi dividido em 11 AEDs (figura 1): Três Lagoas; Vila C; Morumbi; Porto Meira; Jardim São Paulo; Jardim América; Imperatriz; AKLP; Centro; Campos do Iguaçu; e Vila Carimã. Três Lagoas
(AED 1) e Vila Carimã (AED 11) são as maiores em extensão.

\section{Fonte dos dados}

Os dados utilizados foram obtidos no Sistema de Informações de Mortalidade (SIM) do Ministério da Saúde (19). As informações populacionais, socioeconômicas e demográficas e a descrição dos setores censitários e das AEDs foram disponibilizadas em formato digital pelo IBGE. A base cartográfica do município foi disponibilizada pela Prefeitura de Foz do Iguaçu, por meio magnético, no formato DWG $\left(\right.$ AutoCad $^{\odot}$ ), e posteriormente convertida para o formato SHP (shapefile) através do software ArcGIS ${ }^{\circledR}$ versão 9.2 (Environmental Systems Research Institute, Inc., Redlands, EUA).

Para a caracterização do perfil dos homicídios, foram avaliadas a variável dependente (mortalidade por homicídios) e as variáveis independentes (sociodemográficas). Esses dados foram correlacionados com os locais de residência e de ocorrência do evento, em função das AEDs e do contingente populacional. A seleção dos casos de homicídio seguiu a Décima Revisão da Classificação Estatística Internacional de Doenças e Problemas Relacionados à Saúde (CID10) (20), sendo selecionados os códigos X85 a Y09.

Utilizou-se o estimador bayesiano empírico espacial a fim de minimizar possíveis instabilidades da variância da taxa de mortalidade por AED. Em seguida, foi obtida a taxa de mortalidade espe-

FIGURA 1. Localização do Município de Foz do Iguaçu e áreas de expansão demográfica, Brasil, $2009^{a}$

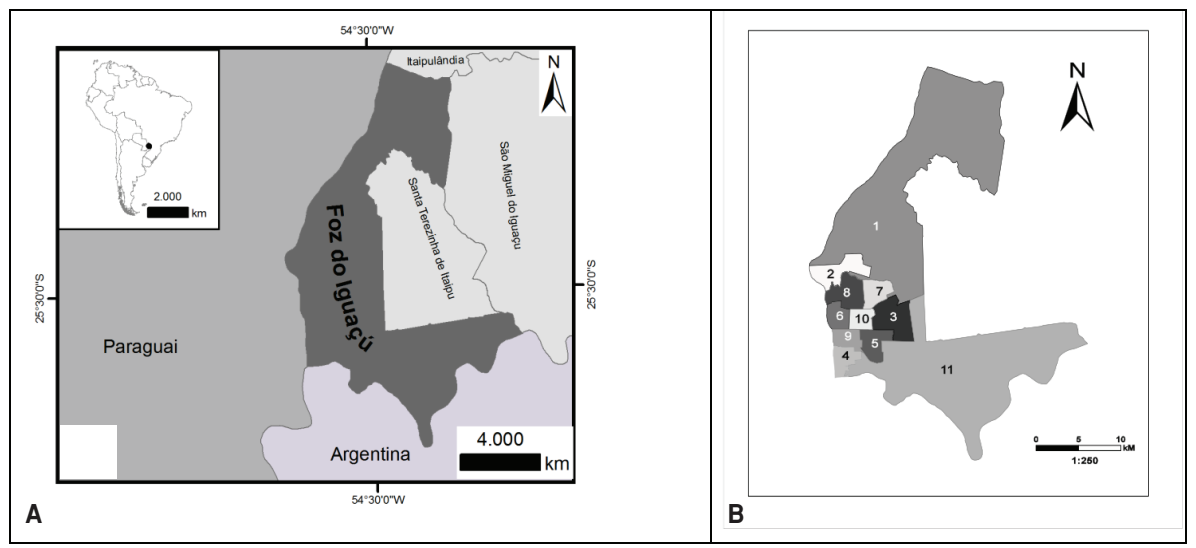

a Os mapas mostram A) o Município de Foz do Iguaçu, localizado no extremo oeste do Estado do Paraná, Região Sul do Brasil (inserto), na tríplice fronteira (Brasil, Paraguai e Argentina); e B) áreas de expansão demográfica (AEDs) em Foz do Iguaçu: 1) Três Lagoas; 2) Vila C; 3) Morumbi; 4) Porto Meira; 5) Jardim São Paulo; 6) Jardim América; 7) Imperatriz; 8) AKLP; 9) Centro; 10) Campos do Iguaçu; 11) Vila Carimã. 
cífica (TME) por 100000 jovens. Analisaram-se também 14 indicadores socioeconômicos e demográficos por AED (número de habitantes, quatro categorias de domicílio, quatro categorias de renda, três categorias de escolaridade e duas categorias de ocupação) para caracterizar as diferentes áreas e a sua relação com o evento homicídio.

\section{Análise espacial}

Primeiramente, foram excluídos 51 óbitos do sexo feminino, por representarem apenas 5,35\% do total dos homicídios, e 29 óbitos do sexo masculino, devido à não localização dos endereços de residência ou ocorrência, registrados como ignorados no SIM. Assim, de 953 mortes juvenis no período de 2000 a 2007, foram analisadas 873 mortes de jovens, distribuídas nas 11 AEDs de Foz do Iguaçu. Por não se dispor da localização exata dos homicídios, mas sim de um valor por área, aplicaram-se técnicas de análise espacial de dados agregados por áreas $(21,22)$.

Utilizou-se a análise exploratória de dados espaciais (AEDE) para a determinação das medidas de autocorrelação espacial global e autocorrelação espacial local (clusters) (23), através do software GeoDa versão 0.9.5-i (Spatial Analysis Laboratory, University of Illinois at Urbana-Champaign, Urbana, EUA) (24).

Para avaliar a existência de autocorrelação espacial, foi definida, previamente, a matriz de peso espacial $-W$, que permite mensurar a associação não aleatória entre o valor de uma variável em uma dada unidade geográfica com o valor da variável nas unidades vizinhas. Neste estudo, utilizou-se a matriz do tipo Queen, que considera todos os vizinhos que tenham fronteira geográfica comum (23).

Calculou-se a autocorrelação espacial considerando as TMEs, os percentuais de importação dos homicídios e os indicadores socioeconômicos e demográficos das AEDs, utilizando-se o índice (I) de Moran global (univariado e bivariado), que mede a autocorrelação espacial ponderada por matriz de vizinhança, indicando se os valores de uma determinada região são ou não semelhantes aos das outras regiões vizinhas (25). $\mathrm{O}$ índice de Moran varia entre -1 e +1 . Valores maiores ou menores que o valor de I de Moran esperado [E (I) $=-1 /(n-1)]$ indicam uma autocorrelação positiva ou negativa, respectivamente. Um valor de I de Moran de 0 (zero) representa a hipótese de independência espacial.

Os valores positivos do I de Moran (entre 0 e +1) indicam autocorrelação espacial positiva (direta), isto é, as áreas estão cercadas por áreas com valores semelhantes (altos ou baixos); os valores negativos do índice I de Moran (entre $0 \mathrm{e}$ -1) representam autocorrelação espacial negativa (inversa), ou seja, as áreas estão circundadas por áreas com valores diferentes (por exemplo, uma área com índice de Moran alto circundada por áreas com índice baixo e vice-versa) (23-25).

Uma limitação do I de Moran global é que ele pode ocultar padrões locais de associação espacial, uma vez que um valor negativo de I de Moran global não indica necessariamente ausência de correlação espacial em nível local (25). Para identificar padrões de associação espacial significativos e específicos a cada área analisada, utilizaram-se indicadores locais de associação espacial (local indicators of spatial association, LISA). Os LISA permitem observar a existência de agrupamentos espaciais (clusters), locais com valores altos ou baixos, e identificar as regiões que mais contribuem para a existência de autocorrelação espacial (25).

Foram gerados mapas coropléticos com os valores das TMEs, percentual de homicídios segundo jovens residentes, jovens não residentes (AED importadora) e jovens que residiam em uma determinada AED e morreram em outra (AED exportadora). Esses valores foram divididos por intervalos de classe e agregados em faixas de desvio padrão em relação à média. Os coeficientes de autocorrelação espacial global e local foram considerados significativos para $P<0,05$. Esses devem ser considerados como níveis de pseudossignificância, ou seja, a sua significância deve ser confirmada pela redistribuição simulada de valores entre as áreas estudadas (teste de permutação) (26).

O estudo foi aprovado pelo Comitê Permanente de Pesquisa em Seres Humanos da Universidade Estadual de Maringá (COPEP-UEM) (parecer 276/2008).

\section{RESULTADOS}

No período estudado, foram registrados 953 homicídios de jovens, com predominância acentuada do sexo mas-
TABELA 1. Distribuição da mortalidade por homicídio em jovens de 15 a 24 anos segundo variáveis sociodemográficas e arma utilizada, Foz do Iguaçu (PR), Brasil, 2000 a 2007

\begin{tabular}{lrr}
\hline Variáveis/categorias & No. & \multicolumn{1}{c}{$\%$} \\
\hline Sexo & & \\
$\quad$ Masculino & 902 & 94,65 \\
$\quad$ Feminino & 51 & 5,35 \\
Faixa etária & 238 & 24,97 \\
15 a 17 anos & 362 & 37,99 \\
18 a 20 anos & 353 & 37,04 \\
$\geq 21$ anos & & \\
Escolaridade & 21 & 2,20 \\
$\quad$ Nenhuma & 294 & 30,85 \\
1 a 3 anos & 350 & 36,73 \\
4 a 7 anos & 228 & 23,92 \\
8 a 11 anos & 34 & 3,57 \\
$\geq 12$ anos & 26 & 2,73 \\
Ignorado & & \\
Cor/raça & 658 & 69,05 \\
$\quad$ Branco & 295 & 30,95 \\
Negro & & \\
Arma utilizada & 901 & 94,55 \\
Arma de fogo & 47 & 4,93 \\
Arma branca & 5 & 0,52 \\
Agressão física & & \\
\hline Fonte: Governo do Estado
\end{tabular}

Fonte: Governo do Estado do Paraná, 9a Regional de Saúde, Secretaria de Saúde do Estado do Paraná, Seção de Vigilância em Saúde (19).

culino, de $94,65 \%$. A idade das vítimas foi de 19,55 $\pm 2,64$ anos. Em relação à escolaridade, $69,78 \%$ das vítimas não chegaram a completar 8 anos de estudo.

Quanto à variável cor/raça, 69,05\% eram brancos, enquanto 30,95\% eram negros. No caso dessa variável, à cor branca somou-se a cor amarela, e à cor negra, a cor parda. Entretanto, levando em conta a estimativa populacional de 42293 jovens de cor branca no município, cerca de 3 vezes mais do que a população negra, estimada em 14035 jovens, o percentual observado de homicídios juvenis foi de 2,1\% entre negros e de 1,6\% entre brancos. A arma de fogo foi utilizada em $94,55 \%$ dos homicídios (tabela 1).

Em seguida, buscou-se identificar a existência de padrões espaciais na distribuição dos homicídios juvenis do sexo masculino nas 11 AEDs do município. A figura 2A apresenta as TMEs dos homicídios por AED de ocorrência. A AED 6 (Jardim América) apresentou a maior taxa - 678/100 000 jovens - , seguida da AED 11 (Vila Carimã), com coeficiente de mortalidade 616,9/100 000. As duas áreas descritas ficam bem perto das fronteiras brasileiras com os países vizinhos, sendo a AED 6 com o Paraguai e a AED 11 com a Argentina. 
FIGURA 2. Distribuição espacial das taxas de mortalidade específica em 100000 jovens segundo a área de expansão demográfica, Foz do Iguaçu (PR), Brasil, 2000 a 2007 ${ }^{a}$

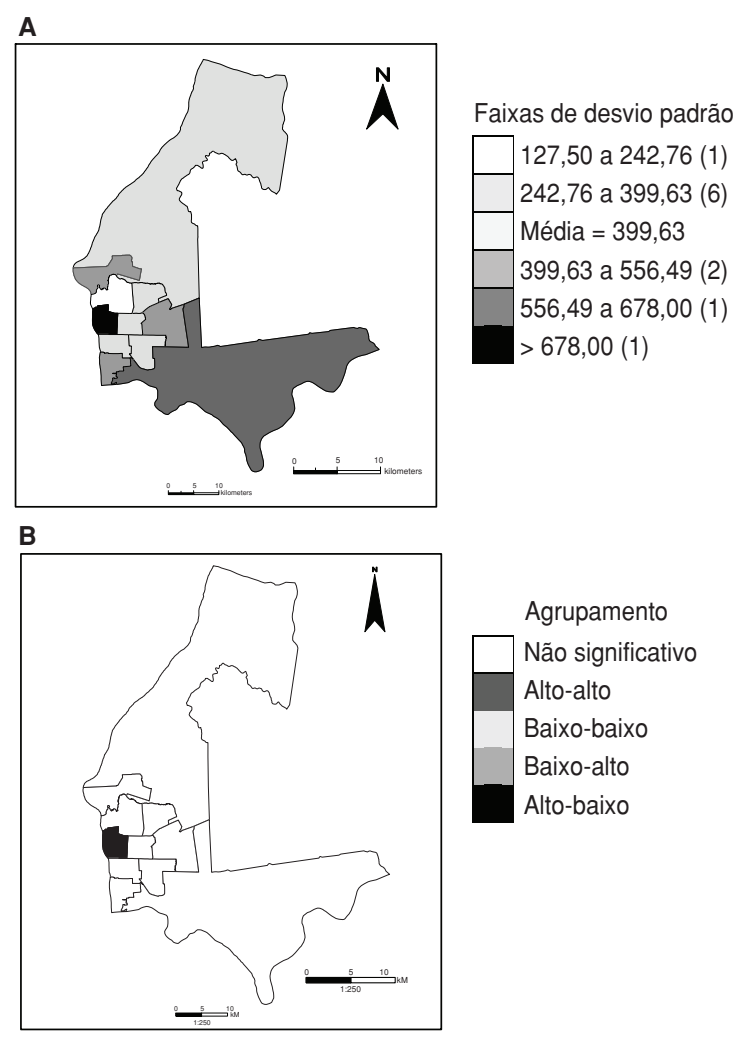

${ }^{a}$ A) Taxa de mortalidade específica segundo local de ocorrência, sendo o desvio padrão o critério de separação dos diferentes grupos (o número de áreas de expansão demográfica em cada faixa foi indicado entre parênteses). B) Mapa de agrupamento espacial (LISA) da taxa de mortalidade específica segundo área de expansão demográfica.

$\mathrm{Na}$ aplicação do I de Moran global univariado, verificou-se a existência de autocorrelação espacial negativa entre as AEDs analisadas quanto ao local de ocorrência para as TMEs por homicídio. Isso foi constatado a partir do valor encontrado na convenção Queen ( $\mathrm{I}=$ $-0,3563, P=0,0250)$, que mostrou que o I estava abaixo do valor esperado $(\mathrm{E}[\mathrm{I}]=-0,1000)$, não sendo detectados agrupamentos por similitude entre as AEDs. Portanto, mesmo se o município apresentasse altas taxas de mortalidade por homicídios em todas as suas regiões, constatou-se que as AEDs com altas taxas de homicídios estavam rodeadas por AEDs com taxas menores, e vice-versa.

A análise da formação de agrupamentos locais (clusters) através da estatística LISA univariada também não identificou nenhum tipo de cluster significativo $(P$ $<0,05)$ das AEDs. Somente apareceu no mapa a AED 6 (Jardim América), localizada perto da Ponte da Amizade, que liga o Brasil ao Paraguai. A AED Jardim Amé- rica apresentou alta taxa de homicídios, mas era cercada por AEDs vizinhas com menores taxas de homicídios, com diferença estatística significativa (figura 2B).

A figura 3 mostra a distribuição espacial dos homicídios de jovens nas AEDs: jovens que morreram na $A E D$ de residência, jovens que morreram em uma AED onde não residiam (AED importadora) e jovens que residiam em uma AED e morreram em outra (AED exportadora). Quando se analisam as ocorrências de jovens residentes (figura 3A), a AED 2 (Vila C) foi a área que apresentou a maior ocorrência de óbitos de jovens residentes nessa área $(89,12 \%)$, seguida da AED 1 (Três Lagoas), com 83,33\%. Constatou-se que, na maioria das AEDs, excetuando-se a AED 9 (Centro) e a AED 10 (Campos do Iguaçu, região da rodoviária interestadual), o maior percentual de óbitos por homicídios juvenis ocorreu na AED de residência da vítima.

Quanto à ocorrência de homicídios de jovens não residentes nas AEDs, per- cebe-se que a AED com maior volume de importação de homicídios foi a AED 9 (Centro), com 60\%, seguida da AED 10 (Campos do Iguaçu), com 51,02\% (figura 3B). Por outro lado, a AED que exportou o maior número de vítimas de homicídios foi a AED 5 (Jardim São Paulo), com $46,15 \%$ de jovens residentes que morreram em outras AEDs, seguida da AED 8 (AKLP), com valor de 43,48\% (figura 3C).

A análise de Moran global bivariada mostrou que, dentre 14 indicadores socioeconômicos, dois se correlacionaram significativamente com a taxa média de homicídio por local de ocorrência $(P<$ $0,05)$. A correlação foi positiva para um deles: pessoas com ocupação no setor formal ( $\mathrm{I}=0,2574, P=0,0310)$. Portanto, quanto maior o número de empregos formais na AED observada, maior era a taxa de homicídios nas AEDs vizinhas. A outra correlação foi negativa: pessoas com ocupação no setor informal ( $\mathrm{I}=$ $-0,2574, P=0,0360)$. Nesse caso, quanto maior o número de empregos informais em uma determinada AED, menor era a taxa de homicídios nas AEDs vizinhas. A análise bivariada do LISA não identificou nenhum tipo de cluster significativo $(P<0,05)$; somente a AED 6 apresentou significância estatística.

Outro ponto importante foi a detecção de autocorrelação espacial positiva do percentual de homicídios segundo a AED "importadora". Também para essa análise foi selecionada a matriz de peso espacial Queen $(I=0,2795, E[I]=-0,1000$, $P=0,0100)$. Para facilitar a visualização das informações com autocorrelação espacial positiva, fez-se uso do LISA cluster map univariado (figura 4A) e bivariado (figura 4B e 4C). Na análise univariada do mapa (figura 4A), identificou-se a presença de cluster alto-alto: as AEDs 6 (Jardim América), 9 (Centro) e 5 (Jardim São Paulo) apresentaram valores altos de importação de homicídios, com vizinhos que também apresentaram valores altos de importação. Essas AEDs estão em regiões próximas do Rio Paraná e da Ponte da Amizade. A AED 4 (Porto Meira) apresentou um padrão espacial baixo-alto, ou seja, teve valores baixos de importação de homicídios enquanto seus vizinhos tiveram valores altos (figura 4A). Observou-se também outra região que apresentou significância estatística, porém sem formação de cluster: a AED 1, que é a região de Três Lagoas, 
FIGURA 3. Percentual de homicídio de jovens residentes e não residentes, Foz do Iguaçu (PR), Brasil, 2000 a $2007^{a}$

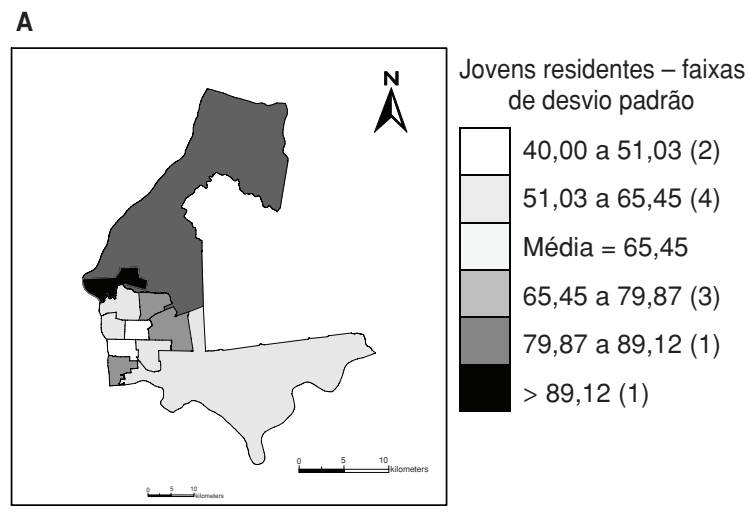

B

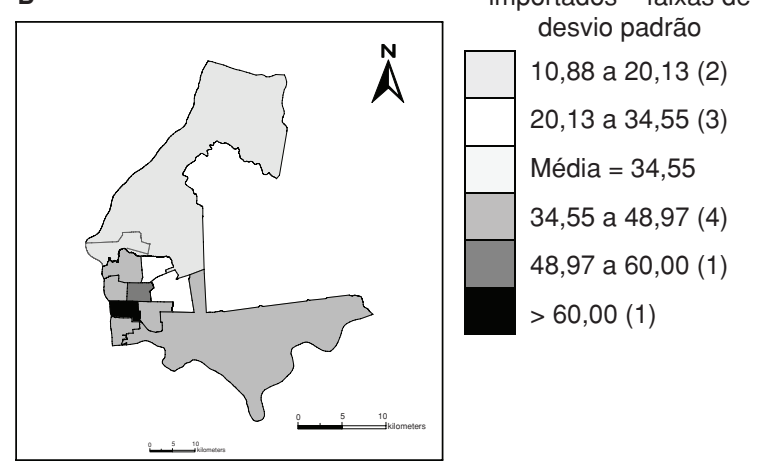

C

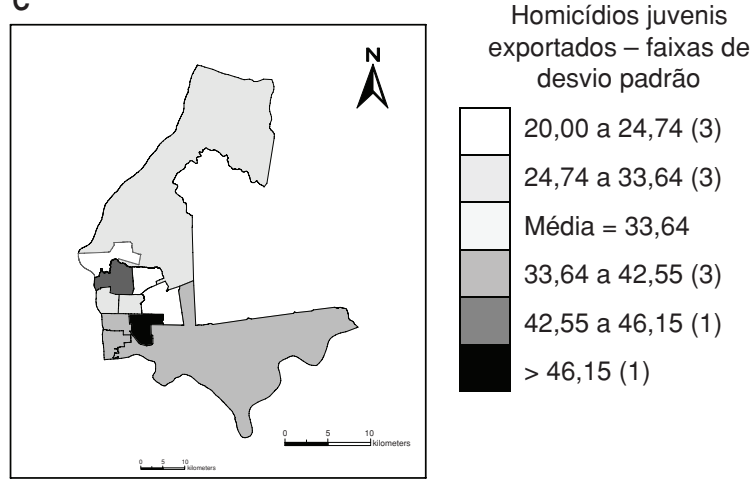

${ }^{\text {a }}$ A) Jovens residentes por área de expansão demográfica (AED); B) jovens não residentes por AED importadora; e C) jovens por AED exportadora. Na legenda dos mapas, o número de AEDs em cada faixa foi indicado entre parênteses.

onde o padrão espacial foi baixo-baixo (valores baixos de importação com vizinhos com valores baixos de importação) (figura 4A).

Houve autocorrelação espacial entre o percentual de importação de homicídios e o grau de ocupação formal e informal nas AEDs de ocorrência. Utilizou-se novamente o I de Moran global bivariado para destacar os indicadores que tiveram associação estatística $(P<0,05)$ com as taxas da AED importadora de homicídios: pessoas ocupadas no setor formal ( $\mathrm{I}=-0,2871, P=0,0180)$, autocorrelação espacial negativa; e pessoas ocupadas no setor informal ( $I=0,2872, P=0,0220)$, autocorrelação espacial positiva.

Na análise LISA bivariada, em relação à variável independente "ocupação no setor formal" (figura 4B), verificou-se a presença de cluster baixo-alto, indicando uma AED com valor baixo de emprego formal cercada por áreas com elevada incidência de importação de homicídios. As AEDs que se destacaram nesse cluster foram: AED 6 (Jardim América), AED 9 (Centro) e AED 5 (Jardim São Paulo). As AEDs 1 (Três Lagoas) e 2 (Vila C) apresentaram um padrão de autocorrelação alto-baixo (áreas com valor alto de emprego formal rodeadas de vizinhos com baixa incidência de importação de homicídios). A AED 4 (Porto Meira) apresentou padrão espacial alto-alto, indicando a existência de alta taxa de emprego formal rodeada de vizinhos com alta incidência de importação de homicídios.

Para a variável independente "ocupação no setor informal" (figura 4C), verificou-se a presença de cluster altoalto, indicando uma AED com valor alto de emprego informal rodeada por áreas com alta incidência de importação de homicídios. As AEDs que se destacaram novamente foram: AED 6 (Jardim América), AED 9 (Centro) e AED 5 (Jardim São Paulo). A AED 4 (Porto Meira) apresentou padrão espacial baixo-alto (valor baixo de emprego informal e vizinhos com alta incidência de importação de homicídios). A AED 1 (Três Lagoas) apresentou padrão de autocorrelação baixo-baixo (área com valor baixo de emprego informal rodeada de vizinhos com baixa incidência de importação de homicídios).

\section{DISCUSSÃO}

O presente estudo identificou um alarmante número de homicídios envolvendo jovens do sexo masculino na faixa etária de 15 a 24 anos em Foz do Iguaçu, município localizado na tríplice fronteira entre Brasil, Paraguai e Argentina. A análise da média anual de homicídios juvenis e da população média de jovens nesse município para o período do estudo mostra um coeficiente de mortalidade de 207,02 em 100000 jovens de 15 a 24 anos. Esse volume de mortes juvenis por homicídio é muito maior do que o volume registrado em grandes cidades brasileiras. Geralmente, o tamanho da população se correlaciona positivamente com os coeficientes de mortalidade, mas fica evidente que isso não ocorre com Foz do Iguaçu $(5,6,27)$.

A distribuição espacial da mortalidade por homicídio, estudada através da TME nas 11 AEDs de Foz do Iguaçu 
FIGURA 4. Análise uni e bivariada de indicadores locais de associação espacial, Foz do Iguaçu (PR), Brasil, 2000 a $2007^{a}$

A

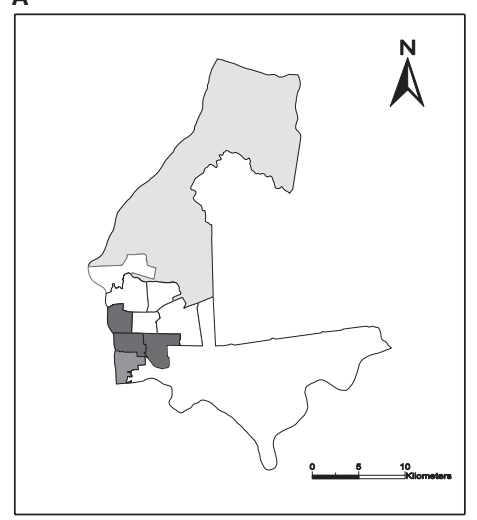

C

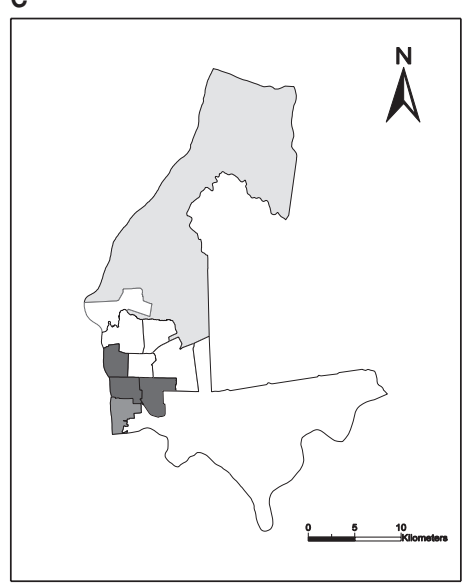

a A) Agrupamento espacial (LISA univariado) do percentual de homicídios segundo área de expansão demográfica importadora. B) Agrupamento espacial (LISA bivariado) do percentual de homicídios segundo área de expansão demográfica importadora e pessoas ocupadas no setor formal. C) Agrupamento espacial (LISA bivariado) do percentual de homicídios segundo pessoas ocupadas no setor informal.

entre os anos 2000 e 2007, evidenciou uma autocorrelação espacial negativa, não sendo detectados agrupamentos de AEDs com TMEs semelhantes. Dos indicadores socioeconômicos analisados, dois se correlacionaram significativamente com a taxa de homicídio por local de ocorrência: a variável "ocupação no setor formal", que indicou que quanto maior o número de empregos formais na AED observada, maiores as taxas de homicídio das AEDs vizinhas; e a variável "ocupação no setor informal", que mostrou o contrário, ou seja, quanto maior o número de empregos informais na AED analisada, menor a taxa de homicídios nas AEDs vizinhas. Nesse caso, a AED 6 (próxima à Ponte da Amizade) se destacou por apresentar alta taxa de ocupação informal e estar rodeada de AEDs vizi-

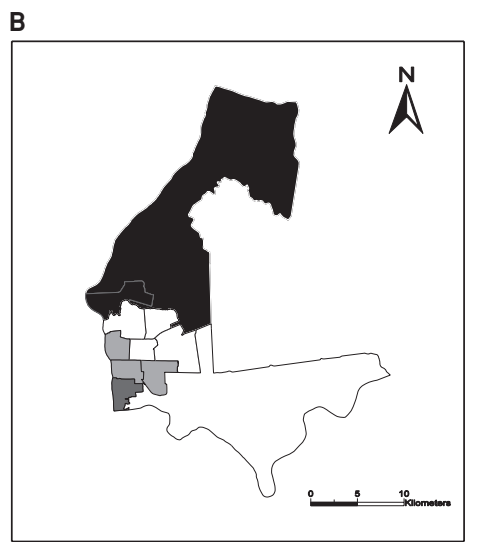

AED importadora (A)

Ocupação formal (B)

Ocupação informal (C)

\begin{tabular}{|l|l|}
\hline Não significativo \\
Alto-alto \\
Baixo-baixo \\
Baixo-alto \\
Alto-baixo
\end{tabular}

nhas com baixas taxas de homicídios, e ainda por apresentar a mais alta taxa de homicídios juvenis do município.

Estudos têm demonstrado associação entre taxas de homicídio e desigualdade socioeconômica. Em Medellín, na Colômbia, os homicídios têm atingido jovens que moram em bairros pobres da cidade, onde predomina o desemprego e o emprego informal associado ao baixo nível educacional (28). Um estudo realizado nos Estados Unidos verificou correlação entre as taxas de homicídio e o grau de distribuição de renda, destacando que houve uma queda do número de homicídios em função da redução do desemprego e da melhoria de renda entre a população mais pobre (29).

Confrontar os dados entre as regiões de residência e de ocorrência é funda- mental para entender o fenômeno da violência (14). Observou-se a presença de associação espacial positiva para importação de homicídios nas AEDs próximas à fronteira com o Paraguai. Ainda, quando a associação com os indicadores socioeconômicos das AEDs foi analisada, destacou-se novamente a presença de correlação espacial com o grau de ocupação formal e informal das respectivas AEDs, dessa vez inversamente, devido à análise por importação. Essas duas variáveis indicaram associação entre a desigualdade socioeconômica (alto grau de ocupação informal e baixo grau de ocupação formal) e a alta importação de homicídios nas AEDs analisadas.

Entender a pobreza social e, em particular, a predominância do nível de desemprego é crucial para a compreensão do fenômeno da violência juvenil em locais específicos (1). Um estudo realizado nos Estados Unidos, nos Condados de Miami-Dade e Broward, observou maiores taxas de homicídios nos grupos populacionais mais pobres e com maior número de indivíduos desempregados (30). No entanto, outros estudos não têm detectado correlação entre a taxa de mortalidade por homicídio e a taxa de pobreza, indicando que a pobreza em si não é um fator determinante, mas reflete, em algumas populações estudadas, desigualdades sociais, possível falta de acesso aos benefícios sociais decorrentes de políticas públicas e insatisfação das necessidades básicas (6).

Foz do Iguaçu apresenta duas grandes linhas de trabalho formal, a usina de Itaipu Binacional, que oferece concursos para profissionais já especializados, e o turismo da tríplice fronteira, que também absorve preferencialmente profissionais qualificados. Essa situação revela um município que não supre as necessidades da população local em relação aos empregos formais. Desse modo, surge como opção o trabalho informal do transporte de mercadorias do Paraguai através da Ponte da Amizade. As ocupações informais são as grandes fontes geradoras de renda para $62,80 \%$ da população iguaçuense (31), o que aponta para um grave problema social.

Através das atividades informais, muitas vezes ilegais, jovens pobres têm encontrado seu espaço na sociedade. Trazer mercadorias do país vizinho não exige um profissional qualificado, com 
experiência, o que facilita a migração de jovens para esse tipo de atividade, deixando-os em situação de risco, na marginalidade econômica e social e aproximando-os da violência fatal.

Uma limitação do presente trabalho foi o fato de não termos investigado possíveis diferenças na distribuição espacial que possam ter ocorrido temporalmente (32). No entanto, esse problema foi minimizado, uma vez que analisamos os homicídios de indivíduos de uma faixa etária específica e apenas do sexo masculino. $\mathrm{O}$ homicídio de mulheres merece pesquisa específica, pois pode apresentar variações espaciais e temporais diferenciadas.

Em resumo, os presentes resultados demonstram que a elevada taxa de mortalidade de jovens em algumas regiões do município, principalmente naquelas próximas à fronteira com o Paraguai, é significativamente influenciada pelo alto grau de ocupação informal, indicando um grave problema social, que é o desemprego associado à falta de qualificação profissional. Assim, conclui-se que as políticas públicas focadas na diminuição da mortalidade juvenil são ainda modestas e impotentes diante da problemática.

\section{REFERÊNCIAS}

1. Legge S. Youth and violence: phenomena and international data. New Dir Youth Dev. 2008;(119):17-24,7-8.

2. World Health Organization. World report on violence and health: summary. Genebra: World Health Organization; 2002. Disponível em: http://www.who.int/violence injury_prevention/violence/world_ report/en/summary_en.pdf Acessado em 08 de setembro de 2011.

3. Reza A, Mercy JA, Krug E. Epidemiology of violent deaths in the world. Inj Prev. 2001;7(2):104-11.

4. Meddings DR, Knox LM, Maddaleno M, Concha-Eastman A, Hoffman JS. World Health Organization's TEACH-VIP: contributing to capacity building for youth violence prevention. Am J Prev Med. 2005;29(5 suppl 2):259-65.

5. Waiselfisz JJ; Rede de Informação Tecnológica Latino-Americana, RITLA; Instituto Sangari; Ministério da Saúde; Ministério da Justiça. Mapa da violência dos municípios brasileiros 2008. Brasília: Ideal Gráfica e Editora; 2008. Disponível em: http://pdba.georgetown. edu/Security/citizensecurity/brazil/docu ments/mapaviolencia.pdf Acessado em 27 de fevereiro de 2012.

6. de Andrade SM, Soares DA, de Souza RK, Matsuo T, de Souza HD. Homicídios de homens de 15 a 29 anos e fatores relacionados no Estado do Paraná, Brasil, 2002/2004. Cienc Saude Coletiva. 2011;16(1):1281-8.

7. Butchart A, Engström K. Sex- and agespecific relations between economic development, economic inequality and homicide rates in people aged 0-24 years: a crosssectional analysis. Bull World Health Organ. 2002;80(10):797-805.

8. Roe-Sepowitz DE. Comparing male and female juveniles charged with homicide: child maltreatment, substance abuse and crime details. J Interpers Violence. 2009;24(4): 601-17.

9. de Souza ER. Masculinidade e violência no Brasil: contribuições para a reflexão no campo da saúde. Cienc Saude Coletiva. 2005;10(1):59-70.

10. Câmara G, Monteiro AM, Fucks SD, Carvalho MS. Análise espacial e geoprocessamento. Em: Druck S, Carvalho MS, Câmara G, Monteiro AM. Análise espacial de dados geográficos. Brasília: EMBRAPA; 2002. Disponível em: http://www.dpi.inpe.br/gilberto/livro/ analise/cap1-intro.pdf Acessado em 20 de março de 2009.

11. Bailey TC, Gatrell AC. Interactive spatial data analysis. Essex: Longman Scientific \& Technical; 1995.

12. de Lima ML, Ximenes RA, de Souza ER, Luna CF, de Albuquerque Mde F. Spatial analysis of socioeconomic determinants of homicide in Brazil. Rev Saude Publica. 2005;39(2):176-82.

13. Bastos Mde J, Pereira Jdos A, Smarzaro DC, Costa EF, Bossanel RC, Oliosa DM, et al. Ecological analysis of accidents and lethal violence in Vitória, Southeastern Brazil. Rev Saude Publica. 2009;43(1):123-32.

14. Santos SM, Barcellos C, Sá Carvalho M. Ecological analysis of the distribution and sociospatial context of homicides in Porto Alegre, Brazil. Health Place. 2006;12(1):38-47.

15. Messner SF, Anselin L. Spatial analyses of homicide with areal data. Em: Goodchild MF, Janelle DG, eds. Spatially integrated social science. Oxford: Oxford University Press; 2004. Pp 127-44.

16. Prefeitura Municipal de Foz do Iguaçu. História da cidade. Disponível em: http://www. pmfi.pr.gov.br/portal2/home_turismo/ historia_cidade.asp Acessado em 25 de maio de 2011.

17. Ministério da Saúde - Departamento de Informática do SUS - Informações de Saúde - Demográficas e Socioeconômicas. Disponível em:http://tabnet.datasus.gov.br/cgi/ deftohtm.exe?ibge/cnv/poppr.def Acessado em 25 de maio de 2011.

18. Instituto Brasileiro de Geografia e Estatística. Censo demográfico 2000, resultados da amostra, agregado de áreas de ponderação (microdados) [CD-ROM]. Rio de Janeiro: IBGE; 2008.

19. Governo do Estado do Paraná, 9a Regional de Saúde, Secretaria de Saúde do Estado do Paraná, Seção de Vigilância em Saúde. Sistema de informações sobre mortalidade. Foz do Iguaçu: 9aㅗ RS/SCVGS/SIMWEB; 2008.

20. Organização Mundial da Saúde. Classificação estatística internacional de doenças e problemas relacionados à saúde, $10^{\mathrm{a}}$ revisão. $8^{\mathrm{a}}$ ed. São Paulo: EDUSP; 2000.

21. Câmara G, Carvalho MS, Cruz OG, Correa V. Análise espacial de áreas. Em: Druck $S$, Carvalho MS, Câmara G, Monteiro AM. Análise espacial de dados geográficos. Brasília: EMBRAPA; 2002. Disponível em: http:// www.dpi.inpe.br/gilberto/livro/analise/ cap5-areas.pdf Acessado em 15 de abril de 2009.

22. Krempi AP. Explorando recursos de estatística espacial para análise da acessibilidade da cidade de Bauru [dissertação]. São Carlos: Escola de Engenharia de São Carlos, Universidade de São Paulo; 2004.

23. Anselin L. Interactive techniques and exploratory spatial analysis. Em: Longley PA, Goodchild MF, Maguire DJ, Rhind DW, eds. Geographical information systems: principles, techniques, management and applications. Wiley: Nova Iorque; 1998. Pp 253-65.

24. Anselin L, Syabri I, Kho Y. GeoDa: an introduction to spatial data analysis. Geogr Anal. 2006;38(1):5-22.

25. Perobelli FS, Haddad EA. Padrões de comércio interestadual no Brasil, 1985 e 1997. Rev Econ Contemp. 2006;10(1):61-88.

26. Campos FG, Barrozo LV, Ruiz T, César CL, Barros MB, Carandina L, et al. Distribuição espacial dos idosos de um município de médio porte do interior paulista segundo algumas características sócio-demográficas e de morbidade. Cad Saude Publica. 2009;25(1):77-86.

27. Kleinschmitt SC. Análise espacial dos homicídios nos municípios do estado do Paraná [dissertação]. Toledo: Universidade Estadual do Oeste do Paraná; 2009.

28. Cardona M, Garcia HI, Giraldo CA, López MV, Suaréz CM, Corcho DC, et al. Homicidios en Medellín, Colombia, entre 1990 y 2002: actores, móviles y circunstancias. Cad Saude Publica. 2005;21(3):840-51.

29. Pickett KE, Mookherjee J, Wilkinson RG. Adolescent birth rates, total homicides, and income inequality in rich countries. Am J Public Health. 2005;95(7):1181-3.

30. Velis E, Shaw G, Whiteman AS. Victim's profile analysis reveals homicide affinity for minorities and the youth. J Inj Violence Res. 2010;2(2):67-74.

31. Instituto de Pesquisa Aplicada (ETHOS). Pesquisa Retrato Foz: Projeto Atitude Foz. Foz do Iguaçu: ETHOS; 2008.

32. Ocanã-Riola R, Mayoral-Cortés JM. Spatiotemporal trends of mortality in small areas of Southern Spain. BMC Public Health. 2010; 10:26.

Manuscrito recebido em 5 de julho de 2011. Aceito em versão revisada em 19 de dezembro de 2011. 
ABSTRACT Objective. To analyze the spatial distribution of juvenile homicide mortality for males in the city of Foz do Iguaçu (state of Paraná), located in the Brazil, Paraguay, and Argentina tri-border area, between 2000 and 2007.

Juvenile homicides and Methods. The data were obtained from the Ministry of Health's Mortality Informainformal work in a Brazilian city in the Brazil, Paraguay, and Argentina tri-border area tion System (SIM) and the Brazilian Geography and Statistics Institute (IBGE). Spatial analysis of 873 deaths distributed in 11 demographic expansion areas (AEDs) was performed using ArcGIS $^{\circledR} 9.2$ and $\mathrm{GeoDa}^{\mathrm{TM}}$ software.

Results. A negative spatial autocorrelation was observed $(I=-0.3563 ; P=0.0250)$, indicating high, but dissimilar, homicide rates in all AEDs. Of 14 socioeconomic indicators analyzed, a negative $(I=-0.2574 ; P=0.0360)$ and positive $(I=0.2574 ; P=0.0310)$ spatial autocorrelation was recorded for informal and formal work, respectively, indicating that the higher the number of informal jobs in a specific AED, the lower the homicide rate in neighboring AEDs. We identified a high rate of juvenile homicides, informal jobs, and importation of juvenile homicide victims from other AEDs into AED 6, located near the Paraguayan border.

Conclusions. The high rate of juvenile homicides in some areas of Foz do Iguaçu is influenced by the high rate of informal occupations. This points to a serious social problem; namely, unemployment associated with lack of professional training.

Key words Violence; homicide; adolescent; young adult; residence characteristics; Brazil. 